\title{
ARTICLE OPEN Superior lattice thermal conductance of single-layer borophene
}

\author{
Hangbo Zhou ${ }^{1}$, Yongqing Cai ${ }^{1}$, Gang Zhang $^{1}$ and Yong-Wei Zhang ${ }^{1}$
}

By way of the non-equilibrium Green's function simulations and first-principles calculations, we report that borophene, a single layer of boron atoms that was fabricated recently, possesses an extraordinarily high lattice thermal conductance in the ballistic transport regime, which even exceeds graphene. In addition to the obvious reasons of light mass and strong bonding of boron atoms, the superior thermal conductance is mainly rooted in its strong structural anisotropy and unusual phonon transmission. For low-frequency phonons, the phonon transmission within borophene is nearly isotropic, similar to that of graphene. For highfrequency phonons, however, the transmission is one-dimensional, that is, all the phonons travel in one direction, giving rise to its ultra-high thermal conductance. The present study suggests that borophene is promising for applications in efficient heat dissipation and thermal management, and also an ideal material for revealing fundamentals of dimensionality effect on phonon transport in ballistic regime.

npj 2D Materials and Applications (2017)1:14 ; doi:10.1038/s41699-017-0018-2

\section{INTRODUCTION}

Thermal transport is known to be one of the major forms of energy transfer in nature. The well-known Fourier's law, a pioneering work by Fourier in 1820s, is a milestone in the study of thermal conduction. Over the past few decades, due to the fast development of nanotechnology, and the rapid rise of novel concepts in manipulating and controlling heat energy transfer, ${ }^{1}$ the understanding on the phonon-derived thermal transport in low-dimensional systems has progressed at a remarkable pace. With regard to thermodynamics and thermal transport properties, carbon-based materials are unique in the sense that carbon atoms can form strong covalent bonds with $\mathrm{sp}, \mathrm{sp}^{2}$, and $\mathrm{sp}^{3}$ hybridizations. Owing to the strong covalent bonding, carbon allotropes, including three-dimensional diamond, two-dimensional (2D) graphene, and quasi-one-dimensional carbon nanotubes, have attracted much interest due to their stability and various fascinating properties. ${ }^{2}$ Although the electronic properties of these carbon allotropes are notably different, for instance, $\mathrm{sp}^{3}$ diamond is a wide band gap insulator while $\mathrm{sp}^{2}$ graphene is a semimetal with extraordinarily high electron mobility, all of them have one common characteristic, i.e., the ultra-high thermal conductivity. $^{3-9}$ For instance, diamond possesses the highest known lattice thermal conductivity in three-dimensional materials. $^{10,} 11$ In terms of nanostructures, carbon nanotube has a thermal conductivity higher than $3000 \mathrm{~W} / \mathrm{Km}^{12}{ }^{13}$ which is the highest among all the one-dimensional materials. In the family of 2D materials, graphene possesses the highest reported thermal conductivity at room temperature, ${ }^{14-18}$ which is $2-3$ orders higher than that of other $2 \mathrm{D}$ materials, such as $\mathrm{MoS}_{2}{ }^{19-25}$ and phosphorene. ${ }^{26-31}$

The underlying reasons for the highest thermal conductivity of carbon-based materials are mainly due to their light atomic mass, short bond length, and strong covalent bonds between carbon atoms. Then, an interesting question arises naturally: Is there any material that its thermal conduction is even better than its carbon counterpart in the same dimension? Possible candidates could be a material made from the element sitting before carbon in the periodic table, and yet possessing comparable or even stronger bond strength. However, hydrogen is in gas phase. Lithium and beryllium are in the form of metallic bonds at room temperature, whose bond strengths are much weaker than covalent bond and thus their lattice thermal conductance is expected to be lower. Therefore, boron is the only possible choice since it has the potential to form strong covalent bonds. However, the three-dimensional structures of boron are either amorphous or polymorphous, thus significantly limiting its thermal conduction.

Recently, a 2D sheet of boron atoms, called borophene, has attracted a plenty of research interest. ${ }^{32-34}$ Remarkably, monolayer borophene has been grown on metal surface ${ }^{35,36}$ and it has been shown that it is stable against oxidization. ${ }^{36}$ Undoubtedly, the experimental realization of borophene raises the hope that it might serve as a high-speed pathway for phonon transport, due to its short covalent bonds and light atomic mass. Although the thermal conductivity ${ }^{37,} 38$ and thermal conductance ${ }^{39}$ of borophene have been explored, the ballistic phonon transport mechanisms and properties of borophene are still largely unknown, and thus a solid understanding is needed in order to provide guidance for the exploration of its applications.

In this work, by using first-principles calculations and nonequilibrium Green's function (NEGF) technique, we investigate the lattice thermal transport properties of borophene for both hexagonal model and $\beta 12$ model. It is found that the thermal conductance of borophene is highly anisotropic. At room temperature, the lowest thermal conductance is $62 \%$ of the highest one in the hexagonal model, while it is only $30 \%$ in the $\beta 12$ model. Remarkably, the highest thermal conductance at room temperature reaches $7.87 \mathrm{nW} /\left(\mathrm{Knm}^{2}\right)$ and $10.97 \mathrm{nW} /\left(\mathrm{Knm}^{2}\right)$ for hexagonal and $\beta 12$ structures, respectively, which are obviously higher than that of graphene.

\footnotetext{
${ }^{1}$ Institute of High Performance Computing, A*STAR, Singapore 138632, Singapore

Correspondence: Gang Zhang (zhangg@ihpc.a-star.edu.sg)

Hangbo Zhou and Yongqing Cai contributed equally to this work.
}

Received: 20 December 2016 Revised: 16 May 2017 Accepted: 30 May 2017

Published online: 19 June 2017 


\section{RESULTS AND DISCUSSIONS}

Phonon dispersion relation of hexagonal borophene

The lattice structure of hexagonal borophene shown in Fig. 1a is the typical monolayer hexagonal boron sheet, which has been reported experimentally. ${ }^{35,} 36$ In this structure, two planes of rectangular lattice form an out-of-plane buckled structure, with the buckling height of $0.89 \AA$. The lattice constants are $1.612 \AA$ in the $x$-direction (the ridge direction) and $2.869 \AA$ in the $y$-direction (the across-ridge direction), respectively. By using density functional perturbation theory (DFPT), we obtained the interatomic force constant (IFC) and hence the phonon dispersion relation of borophene, which is shown in Fig. 1b. With two atoms in the unit cell, monolayer borophene has three acoustic branches: the inplane transverse acoustic (TA) mode, the longitudinal acoustic (LA) mode, and the out-of-plane acoustic mode, and three optical phonon branches. As shown in Fig. 1b, the LA and TA modes around the zone center along the $y$-direction show a lower group velocity than those along the $x$-direction. In addition, the phonon bands of the optical modes along the $y$-direction are generally more flat than that along the $x$-direction. Such phenomenon implies that the bond strength of borophene is highly anisotropic. It is worth mentioning that there exist slightly imaginary modes near $\Gamma$ point, suggesting that the monolayer borophene is unstable against long wavelength periodic distortions, which is consistent with previously calculated phonon dispersion. ${ }^{35}$ Similar imaginary modes were also observed in graphene nanoribbon. ${ }^{40}$ With supporting by a proper substrate, the instability of the structure may be suppressed. It should be noted, however, that despite this small numerical uncertainty in the thermal conductance at extremely low temperature, such slightly imaginary modes should not significantly affect the accuracy in the thermal conductance calculations. ${ }^{40}$

From Fig. $1 \mathrm{~b}$, it is seen that there is a cut-off frequency of $\sim 1350 \mathrm{~cm}^{-1}$ in the phonon spectrum of borophene, which is
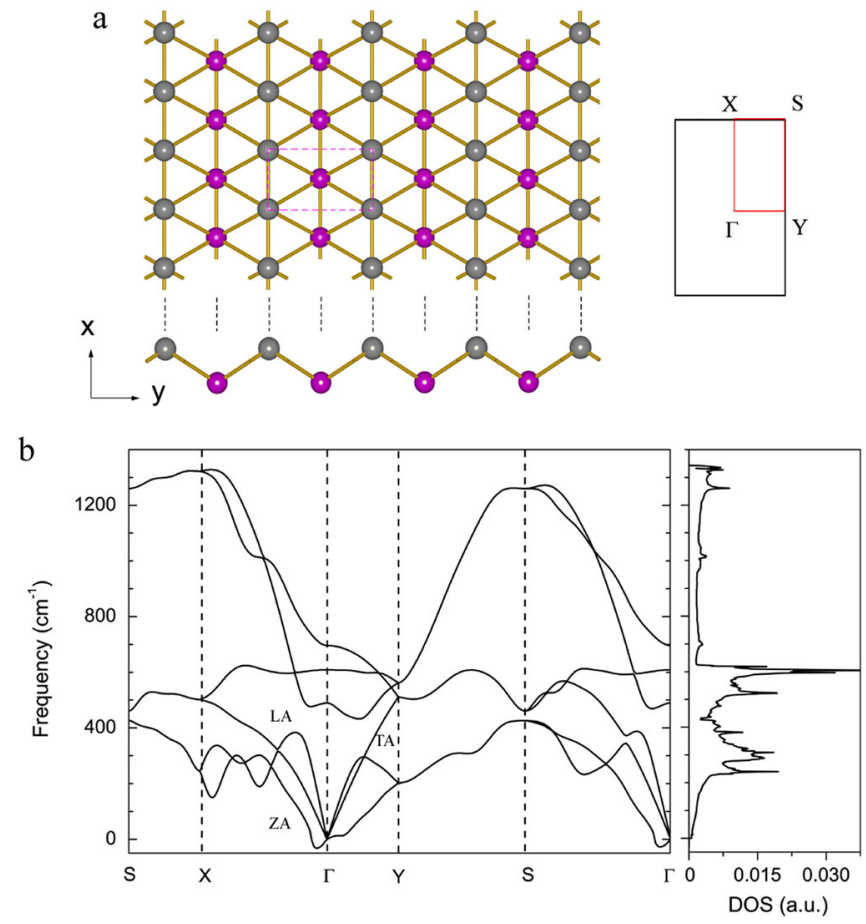

Fig. 1 Structure and phonon dispersion of hexagonal borophene. a The buckled crystal structure of borophene and the dashed lines show the unit cell. The atoms in upper and lower planes are represented by balls in gray and purple, respectively. The inset shows the Brillouin zone. b Phonon dispersion and density of states of borophene based on first-principles calculations comparable to that of graphene $\left(1600 \mathrm{~cm}^{-1}\right){ }^{6}$ This indicates that borophene possesses a strong interatomic bonding. Similar to graphene, the strong covalent bonding in borophene suggests that it has a high Debye temperature. ${ }^{41}$ Therefore, the temperature for the thermal excitation of the highest frequency phonon mode in borophene is comparable to that of graphene, which is about 2000 $\mathrm{K}$. Hence, room temperature $(300 \mathrm{~K})$ can be considered as low temperature with respect to its high Debye temperature, and therefore lattice vibrations should be treated quantum mechanically, in the same way as done in carbon nanotubes and graphene. ${ }^{40,42-45}$

\section{Ultra-high quantum thermal conductance in borophene}

Due to the high Debye temperature $(2000 \mathrm{~K})$, the phonon population is low at room temperature, which suppresses the possibility of phonon-phonon inelastic scattering events. As a result, all lattice vibrations in borophene can be decomposed into normal modes, which are nearly independent of each other. ${ }^{46,} 47$ Furthermore, when the sizes of a material decrease, in particular, when the length scale is comparable or smaller than the phonon mean free path, the inelastic scattering process will be further suppressed. Thus, the thermal transport is quasi-ballistic or ballistic. In this regime, there are many striking phenomena that cannot be explained by phonon diffusion theory, for example, the quantization of thermal conductance ${ }^{48,49}$ and the universal phonon-transmission fluctuation. ${ }^{50}$ Moreover, a study of such a system necessarily requires the incorporation of quantum effects into thermal transport analysis. The NEGF method is able to exactly solve the quantum ballistic transport problems, and therefore it is a powerful tool for studying low-temperature transport properties when non-linear interactions are weak. In fact, it has been widely used to study the heat transport problems in carbon-based materials. ${ }^{40,42-45}$

Here, we employ the NEGF formalism to investigate thermal conductance of borophene. Since a material with a larger width $W$ possesses more phonon transport channels and thus larger thermal conductance, the scaled thermal conductance, defined as the thermal conductance per unit area, is introduced to characterize the thermal transport properties. Herein, the crosssectional area $S$ is defined to be $S=W \times \delta$. For borophene, the buckling height is $0.89 \AA$. Hence, its thickness is chosen as $2.70 \AA$, by adding the diameter of boron atom of $1.81 \AA$.

The thermal conductance per cross-sectional area as a function of temperature along both the $x$-direction and $y$-direction is shown in Fig. 2. The increasing trend of thermal conductance with temperature is general for ballistic transport, since more phonon branches are activated at a higher temperature. ${ }^{40,} 42-45$ As temperature increases, the curve of thermal conductance gradually levels off. Clearly, the room temperature $(300 \mathrm{~K})$ is far below the temperature of $900 \mathrm{k}$, corresponding to the level-off thermal conductance of borophene, further verifying that at room temperature, the number of excited phonons is the major factor that dictates the thermal conductance.

From Fig. 2, it is seen that borophene has a strong anisotropy in thermal conductance. At room temperature, the lattice thermal conductance in the $x$-direction is $7.87 \mathrm{nWK}^{-1} \mathrm{~nm}^{-2}$, while that in the $y$-direction is $4.89 \mathrm{nWK}^{-1} \mathrm{~nm}^{-2}$. Hence, the thermal conductance in the $x$-direction is around two times of that in the $y$-direction. Using the same method, we find that the lattice thermal conductance of graphene at room temperature is $5.09 \mathrm{nWK}^{-1} \mathrm{~nm}^{-2}$ (the green line in Fig. 2), which is consistent with previous results. ${ }^{45,51}$ Remarkably, the quantum thermal conductance in the $x$-direction is $55 \%$ larger than that of graphene. Even in the $y$-direction, the room temperature quantum thermal conductance of borophene is still comparable to that of graphene. We would like to emphasize that this surprising feature of extraordinarily high thermal conductance over graphene has not been observed in other 2D materials. 


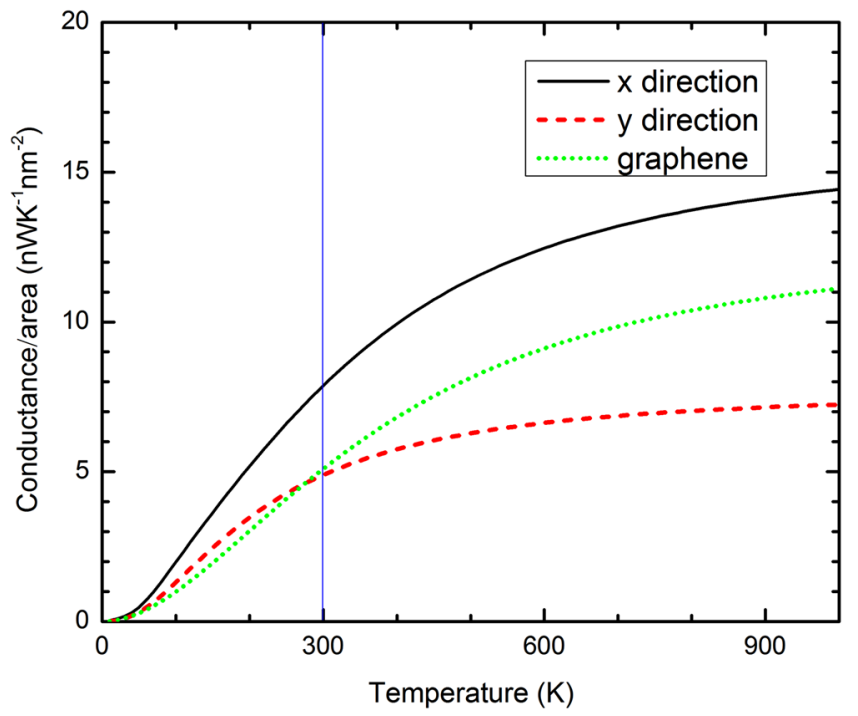

Fig. 2 Thermal conductance of hexagonal borophene. Variations of the thermal conductance per unit cross-sectional area of borophene with temperature in both $x$-direction and $y$-direction. The thermal conductance curve of graphene is also presented for comparison

Conventionally, the atomic mass, interatomic forces, and atomic density are believed to be the major factors affecting the lattice thermal conductance. However, these factors cannot explain the much higher thermal conductance of borophene. For instance, the boron atom is only slightly lighter than carbon atom, and the number of atoms per unit area of borophene in the $x$-direction (13.7 atoms $/ \mathrm{nm}^{2}$ ) is even lower than that of graphene ( 13.9 atoms/ $\mathrm{nm}^{2}$ ). Moreover, the Young's module along the $x$-direction of borophene ( $398 \mathrm{Gpa} \mathrm{nm}$ ) is not remarkably different from that of graphene $(340 \mathrm{Gpanm}){ }^{35}$ signifying that the strength of $B-B$ bond and $\mathrm{C}-\mathrm{C}$ bond are comparable. Hence, new mechanisms that lead to the superior quantum thermal conductance of borophene should exist.

\section{Phonon transmission coefficients}

In order to understand the underlying mechanisms, we investigate the transmission coefficients of the phonons. The transmission coefficient is an important quantity in the NEGF formalism in describing the transmission probability of phonon modes. From the transmission coefficients, we can analyze the contribution of each mode to the lattice thermal conductance and identify the underlying origin why borophene possesses such an extraordinarily high thermal conductance that exceeds graphene.

The transmission coefficients of phonons at different frequencies are shown in Fig. 3a. It is seen that in the low-frequency regime (below $700 \mathrm{~cm}^{-1}$ ), the peaks of phonon transmission in borophene are much higher than those of graphene. These peaks are contributed by acoustic phonon modes. In addition, they are present for the transmission coefficients in both $x$-direction and $y$ direction, and the phonon transmission function along the $x$ direction is similar to that along the $y$-direction.

In the high-frequency regime $\left(700 \sim 1400 \mathrm{~cm}^{-1}\right)$, there is a significant difference in the phonon transmission between the $x$ direction and $y$-direction: in the $y$-direction, the transmission almost vanishes, while in the $x$-direction, the transmission remains substantially high. Interestingly, the transmission coefficients in the $x$-direction keep constant in the high-frequency regime, except for a small central oscillation at about $1000 \mathrm{~cm}^{-1}$. Such feature of constant transmission resembles the phonon transmission along a perfect one-dimensional chain. ${ }^{52}$ In principle, this phenomenon should not exist in $2 \mathrm{D}$ systems due to randomness
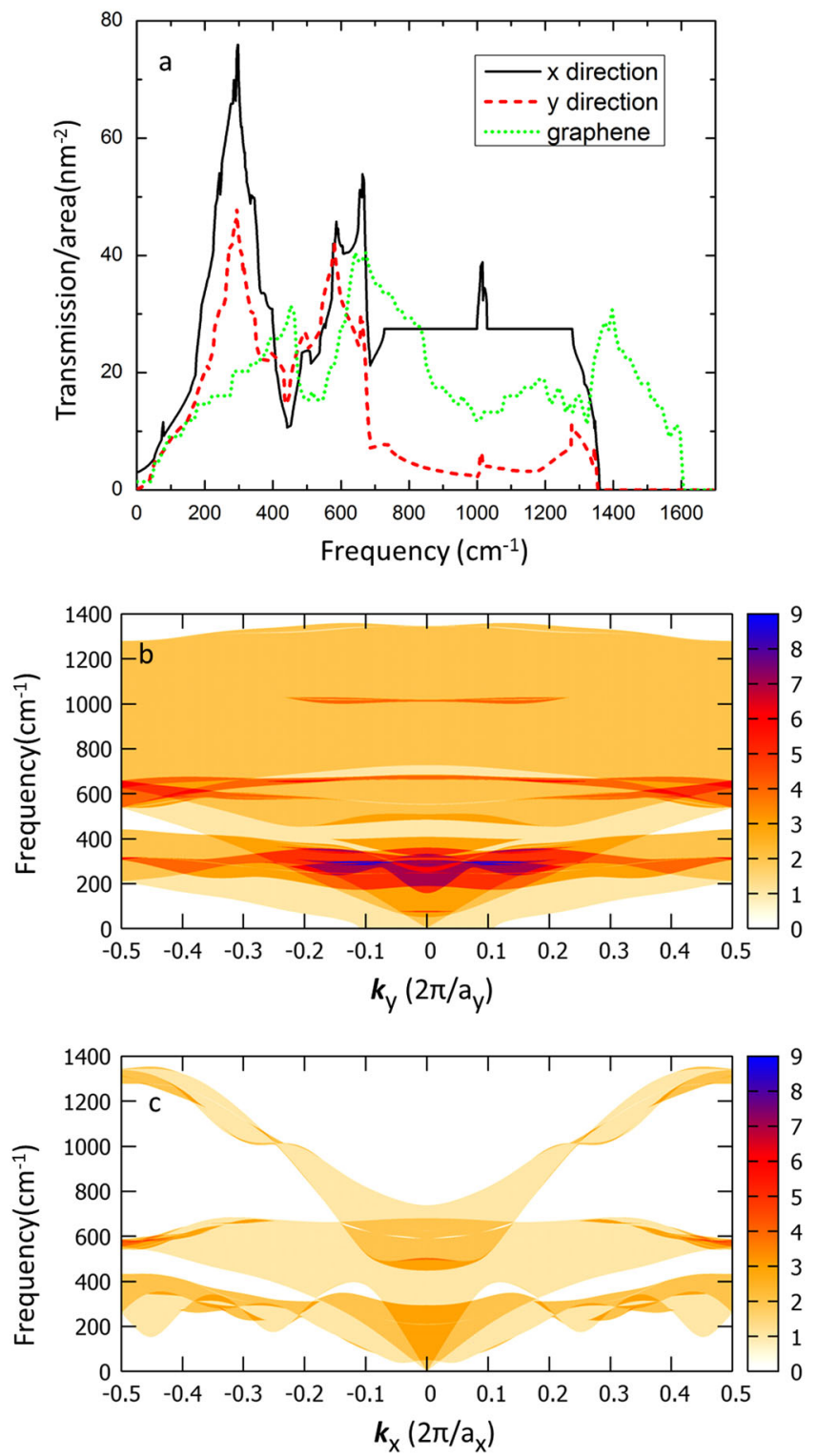

Fig. 3 Transmission coefficient of phonons in borophene. a Plot of transmission coefficient $\mathcal{T}[\boldsymbol{\omega}]$ per unit area against phonon frequency. $\mathbf{b}$ Plot of wave vector-dependent transmission coefficient $\equiv\left[\boldsymbol{\omega}, \boldsymbol{k}_{\boldsymbol{y}}\right]$ of phonons traveling in the $x$-direction against its transverse wave number $k_{\mathrm{y}}$ and frequency $\boldsymbol{\omega}$. c Plot of wave vector-dependent transmission coefficient $\equiv\left[\boldsymbol{\omega}, \boldsymbol{k}_{\boldsymbol{x}}\right]$ of phonons traveling in the $y$-direction against its transverse wave number $k_{x}$ and frequency $\boldsymbol{\omega}$. Here, $a_{\mathrm{x}}$ and $a_{\mathrm{y}}$ are the lattice constants in the $\mathrm{x}$ and $\mathrm{y}$-direction, respectively

of traveling directions of excited phonons and the interference of the phonon modes from different directions. Hence, this unusual behavior in borophene suggests that the traveling phonons in borophene are restricted solely in $x$-direction.

This unusual phonon transmission is further evidenced by the mode-dependence analysis of the transmission coefficients. Figure $3 \mathrm{~b}$ shows the $x$-direction transmission coefficient and Fig. $3 c$ shows the $y$-direction transmission coefficient, which are plotted against phonon frequencies and their respective transverse wave vector. From Fig. $3 b$, it is obvious that in the highfrequency regime there exists a large area with the transmission coefficient $\equiv=2$. This signifies that there exist two phonon modes traveling in the $x$-direction, whose energy persists regardless of the transverse momentum. Such a feature disappears in the 
$y$-direction transmission as shown in Fig. 3c, where the phonon energy is proportional to phonon momentum. Hence, the momentum of phonons is confined along the $x$-direction. Due to the lack of contribution from high-frequency phonon modes, the quantum thermal conductance in the $y$-direction is lower than that along the $x$-direction. As a result, the transport of highfrequency phonon is oriented along the $x$-direction, leading to the quantum thermal conductance along the $x$-direction even higher than that of graphene.

Atomistic origin of ultra-high thermal conductance in borophene In order to understand this unusual behavior of thermal conductance of borophene, we further investigate the wave function and atomistic coupling strength distribution of borophene using density functional theory (DFT) calculations. The electron density distribution from DFT calculation is shown in Fig. 4a. Interestingly, the isosurface plot shows that there is a strong anisotropic distribution in the electronic density: The electron density distribution is dense and one-dimensional along the $B-B$ chain in the $x$-direction, while there are obvious gaps between the chains along the $y$-direction. In general, a denser electron distribution in between the atoms should provide stronger IFCs. From the DFPT calculation, we are able to evaluate the dynamic matrix $D$ at each phonon mode $k$. Then, the real space force-constant matrix $K$ can be obtained by the inverse Fourier transform of the dynamic matrix $D$ at $\boldsymbol{k}$ as following:

$K_{i j}^{\beta}(\boldsymbol{R})=\frac{1}{N_{c}} \sum_{\boldsymbol{k}} e^{i \boldsymbol{k} \cdot \boldsymbol{R}} D_{i j}^{\beta}(\boldsymbol{k})$,

where the $a, \beta$ components correspond to the position vector of the ith, $j$ th atoms in a unit cell, and $\boldsymbol{R}$ is the lattice vector in the real space and $N_{c}$ is the number of unit cells in the crystal. In NEGF calculation, we incorporate the IFC up to the 6th unit cell. For the purpose of illustration, we show in Fig. $4 \mathrm{~b}$ the strength of longitude IFC between nearest neighbor atoms in each direction. The strength of interatomic forces in the $x$-direction is about 30 times of that in the $y$-direction, and 10 times of that between nearest buckling atoms. This implies that borophene has much stronger bonding in the $x$-direction than in the $y$-direction, making borophene like a collection of one-dimensional chains of boron atoms. Consequently, the cut-off frequency of phonon modes in the $y$-direction, which is around $700 \mathrm{~cm}^{-1}$, is much smaller than that in the $x$-direction. As a result, the phonons modes in the $y$ direction (mostly in the low-frequency regime) only interact with the low-frequency modes in the $x$-direction, and they nearly have no interactions with the high-frequency modes in the $x$-direction. Hence, the low-frequency phonon transport exhibits 2D characteristics, while the high-frequency phonons transport shows one-dimensional characteristics. This unique bond strength arrangement leads to the extraordinarily high thermal conductance in the $x$-direction of borophene, which is absent in other 2D materials.

\section{Thermal conductance of $\beta 12$ borophene}

Recently, another atomic structure of single-layer borophene, called $\beta 12$ model, has been reported both experimentally ${ }^{36}$ and theoretically. ${ }^{53}$ The atomic structure of $\beta 12$ model is shown in Fig. 5a. It is seen that no buckling is present in this model, similar to graphene. The lattice constants of structure, obtained from DFT calculation, are $5.062 \AA$ in the $x$-direction and $2.924 \AA$ in the $y$ direction. Figure $5 \mathrm{~b}$ shows the phonon dispersion relation of this structure experienced $1 \%$ tensile strain as suggested in ref. 53 . We find that the cut-off frequency is $1150 \mathrm{~cm}^{-1}$, which is even lower than that of graphene $\left(1600 \mathrm{~cm}^{-1}\right)$. From the phonon density of
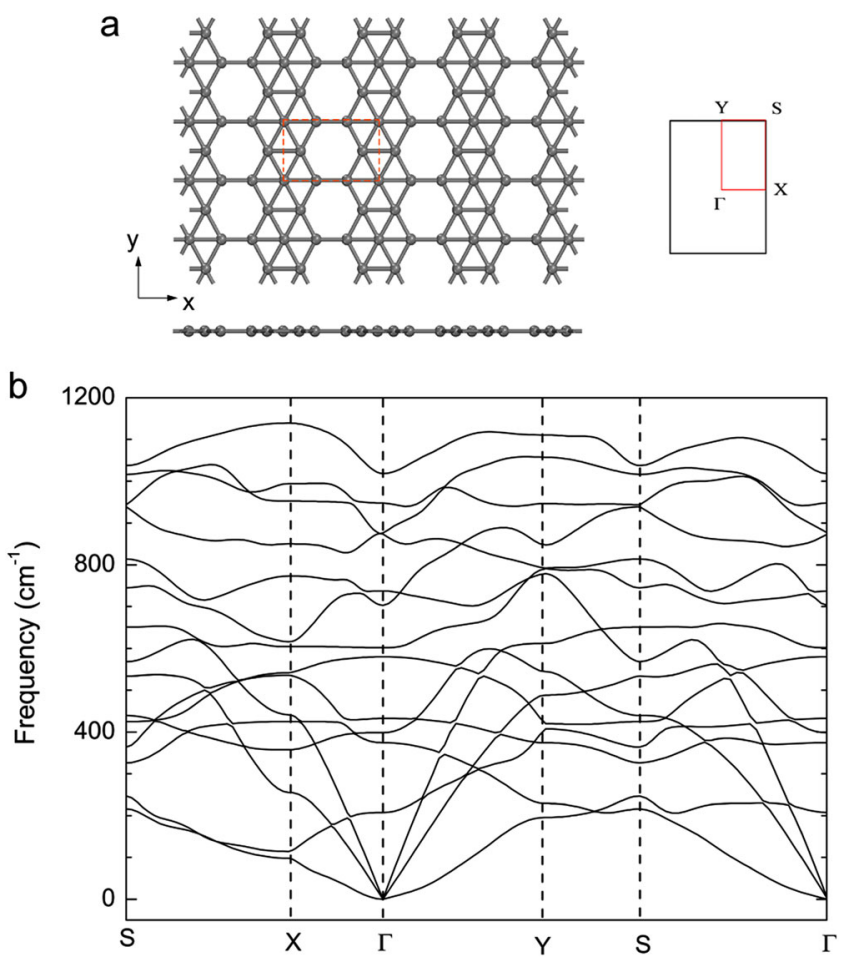

Fig. 5 Structure and phonon dispersion of $\beta 12$ borophene. a Atomic model (top and side views) of the $\beta 12$ borophene sheet. The unit cell is highlighted with dashed lines. The right panel shows the Brillouin zone. b Phonon dispersion of $\beta 12$ borophene based on firstprinciples calculations
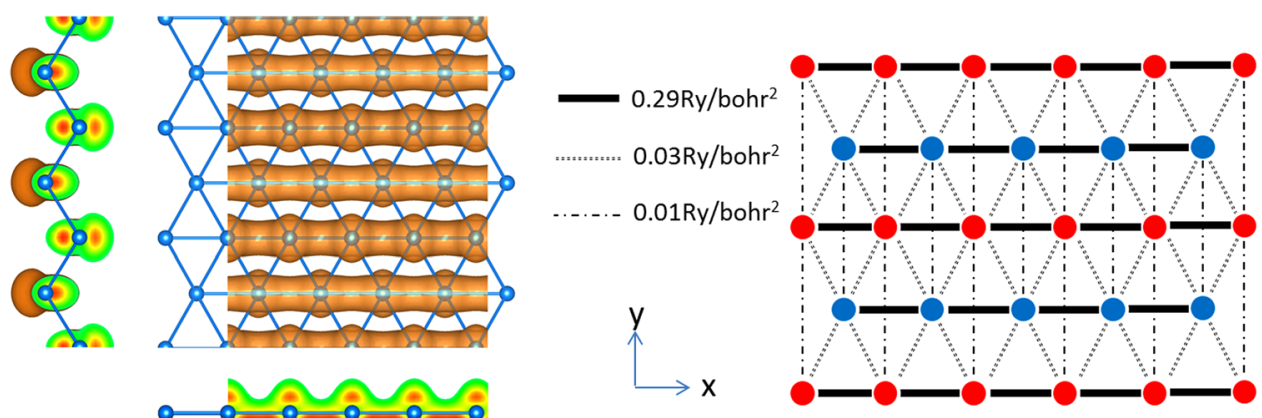

a

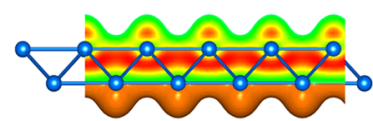

b

Fig. 4 Electron density distribution and force distribution in borophene. a Isosurface plot at $0.15 \AA^{-3}$ for electron density distribution of borophene in both top and side views. $\mathbf{b}$ Top view of interatomic force strength. The red atoms are in the upper plane and the blue ones are in the lower plane. The different types of lines illustrate the longitude IFCs of the nearest neighbor atoms at each direction 
states, we can observe that the low-frequency modes are much denser than high-frequency modes.

By using the same algorithm, we calculated the thermal conductance of $\beta 12$ borophene, as shown in Fig. 6 . Since the thickness of $\beta 12$ borophene has not been reported, we used the diameter of boron atom as the thickness, which is $1.81 \AA$. We find that in $\beta 12$ model, the thermal conductance is highly anisotropic, with the thermal conductance in $x$-direction being much larger than that in $y$-direction. The saturated thermal conductance in $x$ direction is about three times of that in $y$-direction. As shown in Fig. 5a, in $x$-direction, such boron bond forms a boron chain globally. This boron chain greatly concentrates the phonons to travel in the same direction. On the other hand, such boron chain is absent in $y$-direction. In comparison with graphene, a higher thermal conductance is realized in $x$-direction.

In order to analyze the reason of high thermal conductance in $\beta 12$ borophene, transmission function and electron density distribution are presented in Fig. 7. We can find that the transmission function (Fig. 7a) in the $x$-direction is much greater than that in the $y$-direction throughout. It is clear that the main contribution to the thermal conductance is from the lowfrequency phonons (less than $600 \mathrm{~cm}^{-1}$ ). The first peak of

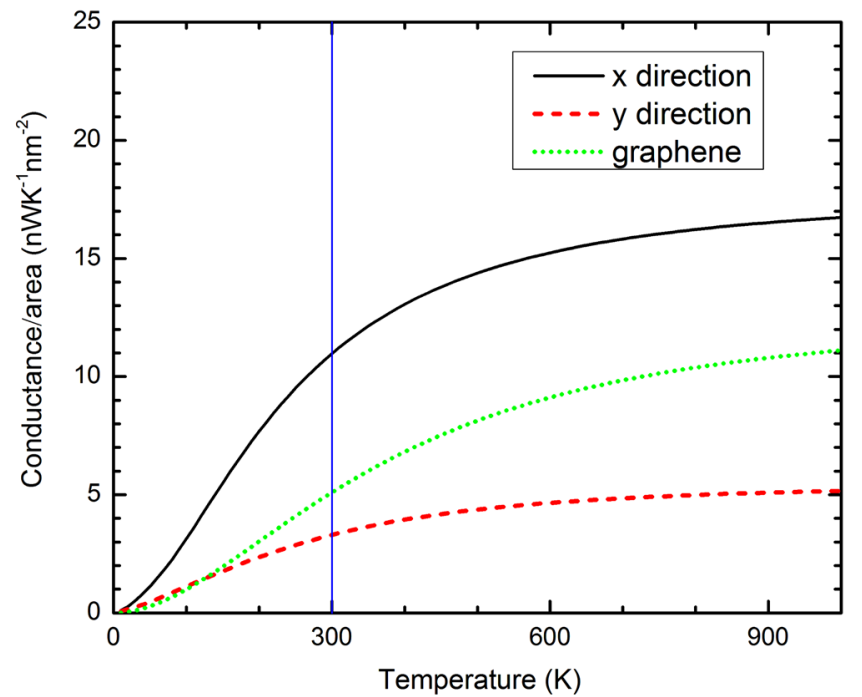

Fig. 6 Temperature-dependent thermal conductance of $\beta 12$ borophene and graphene. The thickness of borophene is taken as the diameter of boron atom. For graphene, the thickness is the interlayer distance $(\delta=3.4 \AA)$ transmission function near $200 \mathrm{~cm}^{-1}$ is mainly due to the acoustic modes, and the major peak at the $400 \mathrm{~cm}^{-1}$ is contributed both from acoustic and optical modes, but not the flexural modes. Hence, this feature is quite different from graphene where the major contribution to thermal conductance is from the flexural modes. Figure $7 \mathrm{~b}$ presents the electron density distribution. Unlike hexagonal borophene, the one-dimensional feature is not present, which is consistent with the absence of one-dimensional behavior in the phonon transmission function. Thus, the mechanism of its high thermal conductance is different from that in hexagonal borophene. In $\beta 12$ borophene, the presence of phonon transmission peak at $400 \mathrm{~cm}^{-1}$ is the major origin of its super-high thermal conductance, indicating that the low-frequency optical modes play a dominated role.

Table 1 summarizes the thermal conductance of borophene and graphene at room temperature. In order to eliminate the thickness effect, we also evaluated the per-length thermal conductance, where the thickness of $2 \mathrm{D}$ material is not divided. It is interesting to find that in $x$-direction, the per-length thermal conductance of both hexagonal and $\beta 12$ borophene sheets is higher than that of graphene, and the $\beta 12$ borophene has a stronger anisotropy than its hexagonal counterpart.

\section{METHODS}

DFT calculation

The IFCs were calculated within the frameworks of DFPT by using the Quantum-Espresso package. ${ }^{54}$ The generalized gradient approximation with the exchange-correlation functional in the Perdew-Burke-Ernzerhof parameterization was adopted together with ultrasoft pseudopotential. For borophene calculation, the first Brillouin zone was sampled with a $48 \times$ $28 \times 1$ and $13 \times 11 \times 1$ Monkhorst-Pack (MP) meshes for electrons and phonons, respectively. Cut-off energies of 40 Ry for plane wave and 600 Ry for electronic density were adopted. For the calculation of graphene, we use MP meshes of $21 \times 21 \times 1$ (electrons) and $8 \times 8 \times 1$ (phonons). The atomic structures and lattice constants were fully relaxed until the forces on each atom became smaller than $1 \times 10^{-6} \mathrm{eV} / A \AA$. In the calculation of phonon dispersion relation, the first step is to check the force constant to valid the acoustic sum rule, which requires that the frequency of acoustic mode at $k=0$ should vanish. This rule is originated from translational invariance, which requires that the net force applied to each atom equals to zero for rigid translation of atoms associated with the acoustic phonons at the zone-center. In this work, the phonon dispersion relation is also calculated using Quantum-Espresso package. ${ }^{54}$

\section{Non-equilibrium Green's function}

By using the DFPT calculation, we obtain the IFCs and hence we can write the Hamiltonian of lattice vibration as a collection of harmonic
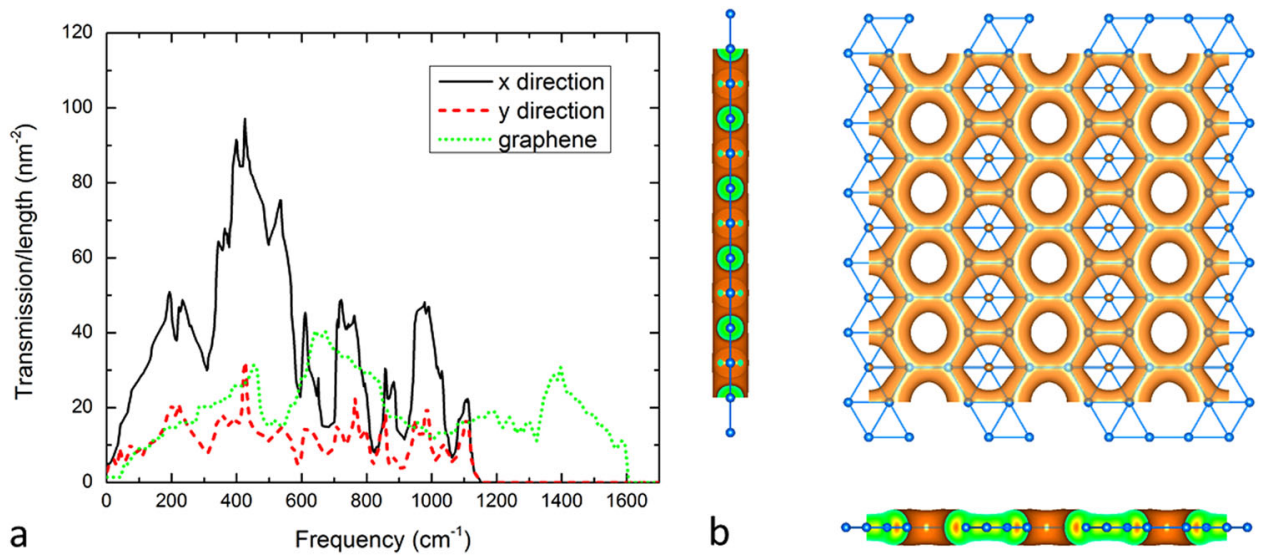

Fig. 7 Phonon transmission and electron density distribution of $\beta 12$ borophene. a Phonon transmission function of $\beta 12$ borophene. b Isosurface plot at $0.15 \AA^{-3}$ for electron density distribution of $\beta 12$ borophene in both top and side views 
Table 1. A summary of thermal conductance of borophene and graphene at $300 \mathrm{~K}$

\begin{tabular}{|c|c|c|c|}
\hline & & $\begin{array}{l}\text { Per-area thermal } \\
\text { conductance }\left(\mathrm{nWK}^{-1} \mathrm{~nm}^{-2}\right)\end{array}$ & $\begin{array}{l}\text { Per-length thermal } \\
\text { conductance }\left(\mathrm{nWK}^{-1} \mathrm{~nm}^{-1}\right)\end{array}$ \\
\hline Borophene (hexagonal model) & $x$-direction & 7.87 & 2.05 \\
\hline \multirow[t]{2}{*}{ Borophene ( $\beta 12$ model) } & $x$-direction & 10.97 & 1.99 \\
\hline & $y$-direction & 3.30 & 0.60 \\
\hline Graphene & Isotropic & 5.10 & 1.71 \\
\hline
\end{tabular}

Note: The per-area thermal conductance is defined as the heat current per temperature gradient per cross-sectional area, where the effect of thickness of $2 \mathrm{D}$ materials is counted. The thickness of borophene is taken as the atomic diameter plus the buckling height. Hence, for the hexagonal model $\delta=2.6 \AA$, and for the $\beta 12$ model $\delta=1.81 \AA$. The per-length thermal conductance is defined as the heat current per temperature gradient per cross-sectional width, where the effect of thickness of $2 \mathrm{D}$ material is not counted

oscillators as:

$$
H=\frac{1}{2 m} p^{T} p+\frac{1}{2} u^{T} K u
$$

where $u$ is a column vector denoting the atomic displacements in all degrees of freedom, and $p$ is the column vector of momentum and conjugate of $u$. The atomic mass is $m=10.8 u$ for boron and $K$ is the force constant matrix obtained from DFPT. We consider that borophene is infinitely large hence only the thermal conductance per cross-sectional area is meaningful, which can be obtained from Landauer formula:

$\sigma / S=k_{B} \int_{0}^{\infty} \frac{d \omega}{2 \pi S} \hbar \omega \mathcal{T}[\omega] \frac{\partial f}{\partial T}$,

where $f[\boldsymbol{\omega}]=1 /\left(e^{\hbar \omega / k_{B} T}-1\right)$ is the Bose-Einstein distribution, $S$ is the cross-sectional area, and $\mathcal{T}[\omega]$ is the total transmission coefficient. By making use of the periodicity in the transverse direction of the transport, we can obtain the transmission coefficients by integrating over the transverse modes:

$$
\mathcal{T}[\omega] / S=\int_{B Z} \frac{d k_{\|}}{2 \pi \delta} \equiv\left[\omega, \boldsymbol{k}_{\|}\right],
$$

where $\delta$ is the thickness of the materials, $\boldsymbol{k}_{\|}$is the wave vector in the transverse direction of the transport, and $\Xi\left[\omega, \boldsymbol{k}_{\|}\right]$is the transmission coefficeints of phonons with energy $\omega$ and transverse wave vector $\boldsymbol{k}_{\|}$, and can be evaluated according to the force constant matrix within the NEGF framework as demonstrated in references. ${ }^{55,56}$

\section{Data availability}

The data that support the findings of this study are available from the corresponding author upon reasonable request.

\section{ACKNOWLEDGEMENTS}

This work was supported in part by a grant from the Science and Engineering Research Council (152-70-00017). The authors gratefully acknowledge the financial support from the Agency for Science, Technology and Research (A*STAR), Singapore, and the use of computing resources at the A*STAR Computational Resource Centre, Singapore.

\section{AUTHOR CONTRIBUTIONS}

H.Z. performed NEGF calculations. Y.C. performed the first-principles calculations. G.Z. and Y.-W.Z. contributed to the discussion and writing of the manuscript. All authors reviewed the manuscript.

\section{ADDITIONAL INFORMATION}

Competing interests: The authors declare no competing financial interests.

Publisher's note: Springer Nature remains neutral with regard to jurisdictional claims in published maps and institutional affiliations.

\section{REFERENCES}

1. Li, N. et al. Colloquium: Phononics: manipulating heat flow with electronic analogs and beyond. Rev. Mod. Phys. 84, 1045-1066 (2012).

2. Chen, G. Size and interface effects on thermal conductivity of superlattices and periodic thin-film structures. J. Heat Transfer 119, 220 (1997).

3. Marconnet, A. M., Panzer, M. A. \& Goodson, K. E. Thermal conduction phenomena in carbon nanotubes and related nanostructured materials. Rev. Mod. Phys. 85, 1295-1326 (2013).

4. Yang, N., Xu, X., Zhang, G. \& Li, B. Thermal transport in nanostructures. AlP Adv. 2, 041410 (2012).

5. Zhang, G. \& Li, B. Impacts of doping on thermal and thermoelectric properties of nanomaterials. Nanoscale 2, 1058 (2010).

6. Balandin, A. A. Thermal properties of graphene and nanostructured carbon materials. Nat. Mater. 10, 569-581 (2011).

7. Sadeghi, M. M., Pettes, M. T. \& Shi, L. Thermal transport in graphene. Solid State Commun. 152, 1321-1330 (2012).

8. Shahil, K. M. F. \& Balandin, A. A. Thermal properties of graphene and multilayer graphene: applications in thermal interface materials. Solid State Commun. 152, 1331-1340 (2012)

9. Pop, E., Varshney, V. \& Roy, A. K. Thermal properties of graphene: fundamentals and applications. MRS Bull. 37, 1273-1281 (2012).

10. Yu, Y., Wu, L. \& Zhi, J. Diamond nanowires: fabrication, structure, properties, and applications. Angew. Chem. Int. Ed. 53, 14326-14351 (2014).

11. Liu, X., Zhang, G. \& Zhang, Y.-W. Surface-engineered nanoscale diamond films enable remarkable enhancement in thermal conductivity and anisotropy. Carbon 94, 760-767 (2015).

12. Berber, S., Kwon, Y.-K. \& Tománek, D. Unusually high thermal conductivity of carbon nanotubes. Phys. Rev. Lett. 84, 4613-4616 (2000).

13. Kim, P., Shi, L., Majumdar, A. \& McEuen, P. L. Thermal transport measurements of individual multiwalled nanotubes. Phys. Rev. Lett. 87, 215502 (2001).

14. Balandin, A. A. et al. Superior thermal conductivity of single-layer graphene. Nano Lett. 8, 902-907 (2008).

15. Chen, S. et al. Thermal conductivity of isotopically modified graphene. Nat. Mater. 11, 203-207 (2012).

16. $\mathrm{Xu}, \mathrm{X}$. et al. Length-dependent thermal conductivity in suspended single-layer graphene. Nat. Commun. 5, 3689 (2014).

17. Seol, J. H. et al. Two-dimensional phonon transport in supported graphene. Science 328, 213-216 (2010).

18. Ghosh, S. et al. Dimensional crossover of thermal transport in few-layer graphene. Nat. Mater. 9, 555-558 (2010).

19. Cai, Y., Lan, J., Zhang, G. \& Zhang, Y.-W. Lattice vibrational modes and phonon thermal conductivity of monolayer MoS 2 . Phys. Rev. B 89, 035438 (2014).

20. Li, W., Carrete, J. \& Mingo, N. Thermal conductivity and phonon linewidths of monolayer $\mathrm{MoS}_{2}$ from first principles. Appl. Phys. Lett. 103, 253103 (2013).

21. Liu, X., Zhang, G., Pei, Q.-X. \& Zhang, Y.-W. Phonon thermal conductivity of monolayer $\mathrm{MoS}_{2}$ sheet and nanoribbons. Appl. Phys. Lett. 103, 133113 (2013).

22. Sahoo, S., Gaur, A. P. S., Ahmadi, M., Guinel, M. J. F. \& Katiyar, R. S. Temperaturedependent Raman studies and thermal conductivity of few-layer $\mathrm{MoS}_{2}$. J. Phys. Chem. C 117, 9042-9047 (2013).

23. Taube, A., Judek, J., Łapińska, A. \& Zdrojek, M. Temperature-dependent thermal properties of supported $\mathrm{MoS}_{2}$ monolayers. ACS Appl. Mater. Interfaces 7, 5061-5065 (2015).

24. Wu, X., Yang, N. \& Luo, T. Unusual isotope effect on thermal transport of single layer molybdenum disulphide. Appl. Phys. Lett. 107, 191907 (2015). 
25. Yan, R. et al. Thermal conductivity of monolayer molybdenum disulfide obtained from temperature-dependent Raman spectroscopy. ACS Nano 8, 986-993 (2014).

26. Lee, S. et al. Anisotropic in-plane thermal conductivity of black phosphorus nanoribbons at temperatures higher than 100 K. Nat. Commun. 6, 8573 (2015).

27. Ong, Z.-Y., Cai, Y., Zhang, G. \& Zhang, Y.-W. Strong thermal transport anisotropy and strain modulation in single-layer phosphorene. J. Phys. Chem. C 118 25272-25277 (2014).

28. Zhu, L., Zhang, G. \& Li, B. Coexistence of size-dependent and size-independent thermal conductivities in phosphorene. Phys. Rev. B 90, 214302 (2014).

29. Zhang, J. et al. Phosphorene nanoribbon as a promising candidate for thermoelectric applications. Sci. Rep. 4, 6452 (2014).

30. Peng, X.-F. \& Chen, K.-Q. Comparison on thermal transport properties of graphene and phosphorene nanoribbons. Sci. Rep. 5, 16215 (2015).

31. Qin, G. et al. Anisotropic intrinsic lattice thermal conductivity of phosphorene from first principles. Phys. Chem. Chem. Phys. 17, 4854-4858 (2015).

32. $\mathrm{Wu}, \mathrm{X}$. et al. Two-dimensional boron monolayer sheets. ACS Nano 6, 7443-7453 (2012).

33. Zhou, X.-F. et al. Semimetallic two-dimensional boron allotrope with massless dirac fermions. Phys. Rev. Lett. 112, 085502 (2014).

34. Li, W. L. et al. The B35 cluster with a double-hexagonal vacancy: a new and more flexible structural motif for borophene. J. Am. Chem. Soc. 136, 12257-12260 (2014).

35. Mannix, A. J. et al. Synthesis of borophenes: anisotropic, two-dimensional boron polymorphs. Science 350, 1513-1516 (2015).

36. Feng, B. et al. Experimental realization of two-dimensional boron sheets. Nat Chem. 8, 563 (2016).

37. Carrete, J. et al. Physically founded phonon dispersions of few-layer materials and the case of borophene. Mater. Res. Lett. 4, 204-211 (2016).

38. Sun, H., Li, Q. \& Wan, X. First-principles study of thermal properties of borophene Phys. Chem. Chem. Phys. 18, 14927-14932 (2016).

39. Tsafack, T. \& Yakobson, B. I. Thermomechanical analysis of two-dimensional boron monolayers. Phys. Rev. B 93, 165434 (2016).

40. Tan, Z. W., Wang, J.-S. \& Gan, C. K. First-principles study of heat transport properties of graphene nanoribbons. Nano Lett. 11, 214-219 (2011).

41. Tohei, T., Kuwabara, A., Oba, F. \& Tanaka, I. Debye temperature and stiffness of carbon and boron nitride polymorphs from first principles calculations. Phys. Rev. B 73, 064304 (2006).

42. Mingo, N. \& Broido, D. A. Carbon nanotube ballistic thermal conductance and its limits. Phys. Rev. Lett. 95, 096105 (2005).

43. Saito, K., Nakamura, J. \& Natori, A. Ballistic thermal conductance of a graphene sheet. Phys. Rev. B 76, 115409 (2007).

44. Xu, Y., Chen, X., Gu, B.-L. \& Duan, W. Intrinsic anisotropy of thermal conductance in graphene nanoribbons. Appl. Phys. Lett. 95, 233116 (2009).
45. Jiang, J.-W., Wang, J.-S. \& Li, B. Thermal conductance of graphene and dimerite. Phys. Rev. B 79, 205418 (2009).

46. Clatterbuck, D. M., Krenn, C. R., Cohen, M. L. \& Morris, J. W. Phonon instabilities and the ideal strength of aluminum. Phys. Rev. Lett. 91, 135501 (2003).

47. Liu, F., Ming, P. \& Li, J. Ab initiocalculation of ideal strength and phonon instability of graphene under tension. Phys. Rev. B 76, 064120 (2007).

48. Rego, L. G. C. \& Kirczenow, G. Quantized thermal conductance of dielectric quantum wires. Phys. Rev. Lett. 81, 232-235 (1998).

49. Schwab, K., Henriksen, E. A., Worlock, J. M. \& Roukes, M. L. Measurement of the quantum of thermal conductance. Nature 404, 974-977 (2000).

50. Yamamoto, T., Sasaoka, K. \& Watanabe, S. Universality and diversity in a phonontransmission histogram of isotope-disordered carbon nanotubes. Phys. Rev. Lett. 106, 215503 (2011)

51. Novoselov, K. S. et al. A roadmap for graphene. Nature 490, 192-200 (2012).

52. Wang, J.-S., Wang, J. \& Zeng, N. Nonequilibrium Green's function approach to mesoscopic thermal transport. Phys. Rev. B 74, 033408 (2006).

53. Zhang, Z. et al. Substrate-induced nanoscale undulations of borophene on silver. Nano Lett. 16, 6622-6627 (2016).

54. Paolo, G. et al. QUANTUM ESPRESSO: a modular and open-source software project for quantum simulations of materials. J. Phys. Condens. Matter 21, 395502 (2009).

55. Wang, J. S., Wang, J. \& Lü, J. T. Quantum thermal transport in nanostructures. Eur. Phys. J. B 62, 381-404 (2008).

56. Zhang, W., Fisher, T. S. \& Mingo, N. Simulation of interfacial phonon transport in $\mathrm{Si}-\mathrm{Ge}$ heterostructures using an atomistic Green's function method. J. Heat Transfer 129, 483 (2007).

Open Access This article is licensed under a Creative Commons Attribution 4.0 International License, which permits use, sharing, adaptation, distribution and reproduction in any medium or format, as long as you give appropriate credit to the original author(s) and the source, provide a link to the Creative Commons license, and indicate if changes were made. The images or other third party material in this article are included in the article's Creative Commons license, unless indicated otherwise in a credit line to the material. If material is not included in the article's Creative Commons license and your intended use is not permitted by statutory regulation or exceeds the permitted use, you will need to obtain permission directly from the copyright holder. To view a copy of this license, visit http://creativecommons. org/licenses/by/4.0/.

(c) The Author(s) 2017 